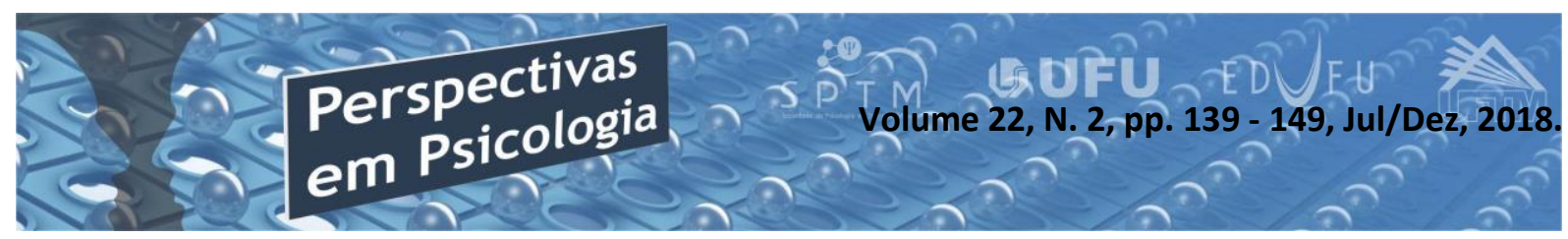

\title{
RELAÇÕES ENTRE ESQUEMAS DE GÊNERO E ESTRESSE EM ADOLESCENTES DO SEXO FEMININO
}

\author{
Sérgio Cardoso Barcelos \\ (CEFET - Araxá-MG) \\ Jorge Luiz da Silva \\ (Universidade de Franca - Franca - SP) \\ Wanderlei Abadio de Oliveira \\ (Faculdade de Filosofia, Ciências e Letras de Ribeirão Preto (FFCLRP/USP) \\ Cléria Maria Lobo Bittar \\ (Universidade de Franca - Franca - SP)
}

\begin{abstract}
Resumo
Esse estudo objetivou identificar diferenças entre os perfis de esquema de gênero e estresse entre adolescentes do sexo feminino. Participaram da pesquisa 93 adolescentes do sexo feminino (idade média 16 anos; desvio padrão 0,8 anos) estudantes do Ensino Médio Profissional de uma cidade do interior de Minas Gerais. A coleta de dados ocorreu por meio da aplicação dos instrumentos: Inventário Feminino dos Esquemas de Gênero do Autoconceito e Escala de Stress para Adolescentes. Análises estatísticas (descritivas, ANOVA e post hoc de Bonferroni) foram realizadas no programa SAS. Os resultados indicaram que $11,8 \%$ do total de participantes foram classificadas com estresse, sendo os maiores percentuais entre as meninas heteroesquemáticas masculinas e as heteroesquemáticas femininas. Do total de participantes, $11,8 \%$ foram classificadas com estresse. Os maiores percentuais localizaram-se nos perfis heteroesquemático masculino $(33,3 \%)$ e heteroesquemático feminino $(33,3 \%)$. Notou-se que o perfil heteroesquemático masculino foi o mais vulnerável para a ocorrência de sintomas interpessoais de estresse, em comparação com os perfis: isoesquemático e heteroesquemático feminino. Concluiu-se que os estereótipos de gênero favorecem a vulnerabilidade individual para o estresse e suas diferentes formas de manifestação.
\end{abstract}

Palavras-chave: Esquemas de gênero; estresse; vulnerabilidade individual.

\section{Abstract \\ Relationships Between Female Sex and Gender Schools and Adolescent Stress}

This study aimed to identify differences between the profiles of gender and stress patterns among female adolescents. A total of 93 female adolescents (mean age 16 years, standard deviation 0.8 years) were enrolled in the Professional High School of a city in the interior of Minas Gerais. Data collection took place through the application of the instruments: Female Inventory of Gender Schemes of Self-Concept and Stress Scale for Adolescents. Statistical analyzes (descriptive, ANOVA and post hoc Bonferroni) were performed in the SAS program. The results indicated that $11.8 \%$ of the total participants were classified as stress, with the highest percentage among male heteroskematics and female heteroskematics. Of the total number of participants, $11.8 \%$ were classified with stress. The highest percentages were found 
in male $(33.3 \%)$ and female heteroskematical profiles $(33.3 \%)$. It was observed that the male heteroskeletal profile was the most vulnerable to the occurrence of interpersonal stress symptoms, in comparison to the female isoesquemic and heteroskematical profiles. It was concluded that gender stereotypes favor individual vulnerability to stress and its different forms of manifestation.

Keywords: Gender schemas; stress; individual vulnerability.

\section{Introdução}

A Teoria dos Esquemas de Gênero (Gender Schema Theory) analisa como as pessoas constroem seu funcionamento cognitivo e comportamental de gênero ao longo do ciclo vital. Os esquemas de gênero fazem parte do autoconceito individual e se relacionam com a masculinidade e a feminilidade. Eles são utilizados pelas pessoas como referências para a categorização de informações, tomadas de decisões e regulação comportamental. Em geral, um indivíduo pode apresentar o esquema masculino ou feminino, ou os dois esquemas, ou ainda serem aesquemáticos. Desse modo, as pessoas podem ser classificadas como heteroesquemáticas (predominância de um dos esquemas: masculino ou feminino) ou isoesquemáticas (simetria entre os esquemas masculino ou feminino (Kulik, 2006; Range \& Jenkins, 2016; Starr \& Zurbriggen, 2017).

Socialmente, traços individualistas (independência, agressividade e racionalidade, por exemplo) são considerados como pertencentes ao esquema masculino, enquanto os traços coletivistas ou expressivos (sensibilidade, empatia, cuidado, por exemplo) são pertinentes ao feminino (Giovani, 2002; Melo \& Giovani, 2010; Melo, Giovani \& Trócolli, 2004; Giovani \& Tamayo, 2005). Isso favorece a construção de estereótipos a partir de experiências passadas e a categorização de pessoas a partir dos próprios processos de internalização sobre como um papel (masculino ou feminino, por exemplo) deve se manifestar socialmente. Sendo que, esses estereótipos se fundamentam em julgamentos e avaliações baseados nos processos perceptivos.

Seguindo uma perspectiva sociocognitiva, a Teoria dos Esquemas de Gênero tem sido amplamente investigada na área da psicologia nos Estados Unidos. Os cinco principais usos da teoria em pesquisa são: desenvolvimento, discriminação/estereótipos, ocupações no mundo do trabalho, populações historicamente marginalizadas, saúde mental e esporte (Starr \& Zurbriggen, 2017). No Brasil a teoria foi divulgada por estudos relacionados ao desenvolvimento 
de um inventário dos esquemas de gênero e sua aplicação entre esportistas (Giovani, 2002; Melo \& Giovani, 2010; Melo, Giovani \& Trócolli, 2004; Giovani \& Tamayo, 2005)

Como os esquemas de gênero influenciam o pensamento, os sentimentos e os comportamentos das pessoas (Starr \& Zurbriggen, 2017), eles podem se associar ao estresse, que constitui uma reação orgânica, com componentes psicofisiológicos que, em geral, possui múltiplas fontes, mas que pode ser desencadeada principalmente por situações (positivas ou negativas) de mudança ou que exigem adaptação (Gonzaga, 2013; Just \& Enumo, 2015; Sadir; Bignotto \& Lipp, 2010). A relação entre esquemas de gênero e estresse pode ocorrer porque homens e mulheres desempenhavam diferentes papéis de gênero, pois a sociedade possui expectativas diferentes sobre o masculino e feminino, o que pode gerar nas pessoas diferentes disposições emocionais e subjetivas relacionadas ao gênero e sua experiência no contexto social (Giovani, 2002; Range \& Jenkins, 2016). Se essas diferentes disposições ocasionarem estresse, ele pode afetar a qualidade de vida e a sensação de bem-estar, impactando na saúde física e mental das pessoas (Gonzaga, 2013; Sadir e outros, 2010).

Segundo a literatura especializada, quando comparadas aos homens, as mulheres possuem maior vulnerabilidade paras os adoecimentos subjetivos ou relacionados à saúde mental, como o estresse (Lee \& Dik, 2017). Existem indicações também de que as diferenças de gênero surgem na adolescência e as meninas tendem a relatarem mais eventos estressores (Mezulis e outros, 2010). Esse cenário revela a importância de se investigar os perfis de esquema de gênero de adolescentes para estabelecer medidas de estresse que possam se relacionar com as questões/expressões de masculinidade e feminilidade. Contudo, uma breve busca bibliográfica nas Bibliotecas Virtuais Scielo e Pepsic, a partir dos descritores estresse (stress) e gênero, estresse (stress) e adolescência, estresse (stress) e adolescente, revelou lacunas na literatura nacional sobre a temática, pois poucos estudos foram identificados.

Além disso, as pesquisas divulgadas não focalizavam, especificamente, a interface entre gênero e estresse na adolescência, mas se relacionavam ao sofrimento psíquico de forma geral (Crivelatti e outros, 2006), a comportamentos de risco dos adolescentes (Carvalho e outros, 2011), ao peso corporal e aos relacionamentos amorosos (Pinto e outros, 2017) como preditores para a ocorrência de estresse, por exemplo. Assim sendo, a presente pesquisa objetivou identificar diferenças entre os perfis de 
esquema de gênero e estresse entre adolescentes. Entender as diferenças entre as variáveis em análise pode ajudar no desenvolvimento de estratégias e intervenções capazes de minimizar o efeito da questão de gênero no desenvolvimento de quadros de estresse.

\section{Método}

\subsection{Participantes}

A amostra do estudo foi composta por 93 adolescentes do sexo feminino que cursavam Ensino Médio Integrado à Educação Profissional no Centro Federal de Educação Tecnológica de Minas Gerais CEFET, localizado na cidade de AraxáMG. As participantes possuíam idade entre 15 e 17 anos, com média de 16 anos $(\mathrm{DP}=0,8$ anos). Elas frequentavam os cursos de Edificações (39,8\%), Mineração (36,6\%), Mecânica (12,9\%) e Eletrônica (10,8\%). Em relação ao nível socioeconômico, a maioria dos estudantes $(62,2 \%)$ pertencia a famílias das classes B1 e B2, que possuem um poder aquisitivo mais elevado, de acordo com o Critério de Classificação Socioeconômica Brasil, o que equivale dizer que arenda familiar total variava entre $R \$ 4.852,00$ e $R \$ 9.254,00$, como $22,3 \%$ do total das famílias brasileiras (ABEP, 2015).

\subsection{Instrumentos}

\subsubsection{Inventário Feminino dos Esquemas de} Gênero do Autoconceito - IFEGA (Giavoni \& Tamayo, 2005). Instrumento de autorrelato destinado à avaliação de esquema de gênero em mulheres e que possibilita a definição de três perfis de gênero: heteroesquemático masculino, heteroesquemático feminino e isoesquemático. O IFEGA é constituído por 75 itens com cinco pontos, sendo 36 referentes à escala masculina e 39 concernentes à escala feminina. Os itens são respondidos em uma Escala Likert de cinco pontos, que variam em intensidade, desde "não se aplica" a "se aplica totalmente". Os fatores que compõem a escala masculina do instrumento são: arrojamento, egocentrismo e negligência. A escala feminina é constituída pelos fatores: sensualidade, inferioridade e ajustamento social. Trata-se de um instrumento que apresenta boa consistência interna para todos os fatores, com alfas de Cronbach variando entre 0,73 e 0,92 .

\subsubsection{Escala de Stress para Adolescentes -} ESA (Tricolli \& Lipp, 2005). A escala é composta por 44 itens e tem como objetivo verificar a existência ou inexistência de estresse. Ela também determina os tipos de 
sintomas predominantes (psicológicos, cognitivos, fisiológicos e interpessoais) e as fases de estresse que adolescentes de 14 a 18 anos se encontram (alerta, resistência, quase-exaustão e exaustão). Os itens são respondidos em uma escala do tipo Likert de cinco pontos, de acordo com a frequência dos sintomas, com variação entre (1) "não sente" a (5) "sente sempre". Também pode variar de acordo com o período em que os adolescentes experimentam as reações apontadas pelos itens, por exemplo: (1) "não ocorreu" a (5) "tem ocorrido nos últimos seis meses". A consistência interna da escala é considerada boa, com Alpha de Cronbach de 0,89.

\subsection{Procedimentos}

Incialmente o projeto de pesquisa foi submetido para avaliação e aprovado pelo Comitê de Ética em Pesquisa da Universidade de Franca (protocolo $\mathrm{n}^{\circ}$ CAAE: 16299813.0.0000.5495). Além disso, em todas as etapas do estudo os pesquisadores seguiram as recomendações da Resolução 466/2012 do Conselho Nacional de Saúde. A escola foi selecionada de forma não probabilística, por acessibilidade. Todas as estudantes matriculadas nos cursos de Edificações, Eletrônica, Mecânica e Mineração foram convidadas a participar do estudo. Para tanto, receberam informações detalhadas sobre os objetivos da pesquisa, sobre como os dados seriam coletados e sobre a liberdade de participação. Não participaram da coleta de dados as estudantes que se recusaram a colaborar com a pesquisa e aquelas que não apresentaram o Termo de Consentimento Livre e Esclarecido assinado por um responsável (TCLE). Os instrumentos de coleta de dados (IFEGA e ESA) foram aplicados em 14 turmas, de maneira coletiva, durante os horários das aulas de Educação Física. A psicóloga da escola auxiliou o pesquisador responsável pelo estudo a aplicar os questionários e a esclarecer as dúvidas das estudantes. A duração média da coleta de dados em cada turma era de aproximadamente 50 minutos.

\subsection{Análise dos dados}

Primeiramente, os dados foram inseridos em uma planilha do programa Excel. Em seguida, foram realizadas as análises estatísticas no programa SAS (versão 9.2). Todas as variáveis do estudo foram descritas em frequência, porcentagem, média e desvio-padrão. Para a definição dos três perfis de esquema de gênero

(Isoesquemático, Heteroesquemático e Heteroesquemático) utilizou-se o método de análises espaciais proposto pelos autores da Escala IFEGA (Giavoni \& Tamayo, 2003). Análises de variância (ANOVA) foram realizadas para 
as variáveis de estresse em relação aos perfis de esquema de gênero. Testes post hoc utilizando a correção de Bonferroni esclareceram as diferenças entre os grupos. Em todas as análises adotou-se um nível de significância de 5\%, p<0,05.
Em relação aos esquemas de gênero, as estudantes da amostra $(n=93)$ foram classificadas como: isoesquemáticas (93,6\%), heteroesquemáticas femininas $(3,2 \%)$ e heteroesquemáticas masculinas $(3,2 \%)$. Os resultados para estresse estão apresentados na Tabela 1.

\section{Resultados}

Tabela 1. Estudantes com estresse de acordo com o perfil de esquema de gênero.

\begin{tabular}{ccccccc}
\hline & \multicolumn{2}{c}{\begin{tabular}{c} 
Isoesquemáticos \\
\cline { 2 - 7 }
\end{tabular}} & $\begin{array}{c}\text { Heteroesquemático } \\
\text { Masculino }\end{array}$ & \multicolumn{3}{c}{$\begin{array}{c}\text { Heteroesquemático } \\
\text { Feminino }\end{array}$} \\
\hline Estresse & & $\%$ & $\mathrm{n}$ & $\%$ & $\mathrm{n}$ & $\%$ \\
Sim & 9 & 10,3 & 1 & 33,3 & 1 & 33,3 \\
Não & 78 & 89,7 & 2 & 66,7 & 2 & 66,7 \\
Total & 87 & 100 & 3 & 100 & 3 & 100 \\
\hline
\end{tabular}

Os resultados da Tabela 1 indicam

que $11,8 \%$ do total de participantes foram classificadas com estresse. Os maiores percentuais de estresse ocorrem para as meninas heteroesquemáticas masculinas
$(33,3 \%)$ e heteroesquemáticas femininas $(33,3 \%)$. A Tabela 2 apresenta as comparações entre os três perfis de esquema de gênero e as variáveis de estresse.

Tabela 2. Diferenças entre os perfis de esquema de gênero e estresse.

\begin{tabular}{lcccccccc}
\hline & \multicolumn{3}{c}{ ISO } & \multicolumn{3}{c}{ HM } & \multicolumn{3}{c}{ HF } & \multicolumn{2}{c}{ Anova } \\
\cline { 2 - 9 } & Média & DP & Média & DP & Média & DP & F & p \\
\hline Sintomas psicológicos & 2,77 & 0,75 & 3,43 & 1,48 & 3,20 & 1,20 & 1,41 & 0,25 \\
Sintomas cognitivos & 2,08 & 0,74 & 2,94 & 1,73 & 2,22 & 0,58 & 1,83 & 0,16 \\
Sintomas fisiológicos & 2,31 & 0,75 & 2,45 & 1,16 & 2,33 & 0,98 & 0,05 & 0,95 \\
Sintomas Interpessoais & 1,98 & 0,76 & 3,33 & 0,81 & 2,00 & 0,20 & 4,65 & 0,01 \\
Estresse total & 2,79 & 0,84 & 3,25 & 1,33 & 3,19 & 1,29 & 0,71 & 0,49 \\
Fase de alerta & 3,12 & 0,93 & 3,47 & 1,58 & 3,60 & 1,24 & 0,53 & 0,58 \\
Fase de resistência & 2,64 & 0,94 & 3,44 & 1,06 & 2,78 & 1,23 & 1,06 & 0,35 \\
Fase de quase exaustão & 2,44 & 0,97 & 2,64 & 0,88 & 2,97 & 1,48 & 0,47 & 0,62 \\
Fase de exaustão & 2,56 & 1,07 & 3,40 & 2,12 & 2,87 & 1,30 & 0,93 & 0,39 \\
\hline \multicolumn{1}{c}{ Nota. *Isoesquemático; **Heteroesquemático Masculino; ***Heteroesquemático Feminino. }
\end{tabular}

Na Tabela 2, a ANOVA revelou não existir diferenças significativas nas médias de sintomas psicológicos, sintomas cognitivos, sintomas fisiológicos, estresse 
total, fase de alerta, fase de resistência, fase de quase exaustão e fase de exaustão. Para sintomas interpessoais, houve diferença estatisticamente significativa entre as médias dos perfis de esquema de gênero entre as meninas isoesquemáticas e as heteroesquemáticas masculinas $(\mathrm{p}=0,003)$, bem como entre as meninas heteroesquemáticas masculinas e heteroesquemáticas femininas $(\mathrm{p}=0,03)$.

\section{Discussão}

Os resultados desse estudo indicaram que a maioria das participantes apresentava perfil de esquema de gênero isoequemático $(93,6 \%)$. Do total de participantes, $11,8 \%$ foram classificadas com estresse. Os maiores percentuais localizaram-se nos perfis heteroesquemático masculino $(33,3 \%)$ e heteroesquemático feminino (33,3\%). Notou-se que o perfil heteroesquemático masculino foi o mais vulnerável para a ocorrência de sintomas interpessoais de estresse, em comparação com os perfis: isoesquemático e heteroesquemático feminino.

No decorrer do desenvolvimento, as crianças e os adolescentes vão internalizando padrões culturais sobre o que se espera do homem e da mulher, o que pode favorecer o surgimento de estereótipos e situações de preconceito e discriminação.
Em termos práticos, há uma classificação baseada em gênero que é imposta e regula como os diferentes comportamentos e maneiras de existir serão relacionados ao homem ou à mulher (Kulik, 2006; Range \& Jenkins, 2016; Starr \& Zurbriggen, 2017). Essa lógica se sustenta na expectativa de adequação de gênero e na rejeição de qualquer manifestação que não coincida o sexo biológico. Essas situações podem precipitar a ocorrência de quadros de estresse ou sua sintomatologia.

Para as mulheres, especificamente, esses aspectos são relevantes na medida em que se compreende que o gênero é um reflexo psicossocial marcado pela ideologia coletiva e pela organização da sociedade. Assim sendo, os traços individualistas (independência, agressividade e racionalidade, por exemplo), considerados como pertencentes ao gênero masculino, podem justificar a maior ocorrência de sintomas de estresse interpessoal para as meninas com perfil do tipo heteroesquemático masculino, pois em suas relações sociais elas agem com características comportamentais do gênero oposto ao seu. O esperado socialmente é que elas apresentassem traços coletivistas ou expressivos (sensibilidade, empatia, cuidado, por exemplo), pertinentes ao feminino (Caputo; Rombaldi \& Silva, 2017). Nessas situações, podem ocorrer nas interações sociais a discriminação de 
gênero, que prediz sofrimento psicológico, ansiedade, estresse, raiva, sintomas somáticos e depressão (Lee \& Dik, 2017; Mezulis e outros, 2010).

É importante destacar que as discussões recentes sobre teorias de gênero colocam dilemas e problemáticas a serem consideradas ao homem ou à mulher (Range \& Jenkins, 2016; Starr \& Zurbriggen, 2017). Percebe-se que, por exemplo, a literatura indica $o$ quanto $o$ desenvolvimento de crianças e adolescentes aesquematicos de gênero favorece a resistência a estereótipos sexistas, consolidados pela sociedade em geral, o que é capaz de diminuir o sofrimento psíquico experimentado por muitas pessoas. $\mathrm{Na}$ contramão dessa perspectiva, por exemplo, muitos livros de entretenimento e maneiras de abordar meninos e meninas na infância proporcionam uma visão não realista do mundo atual, pois auxiliam na definição de estereótipos de gênero, fazendo com que mais crianças sejam esquemáticas em termos de gênero (Fitzpatrick \& Mcpherson, 2010).

Dessa forma, em termos de implicações práticas, as evidências desse estudo acrescentam dados adicionais para pensar o impacto dos esquemas de gênero (construção sociocognitiva relacionada às características compartilhadas por homens e mulheres) na saúde dos adolescentes. Os dados devem ser considerados por equipes de saúde e profissionais para pensar estratégias e intervenções contra o estresse ou que favoreçam maior qualidade de vida e bem-estar entre adolescentes. Essas ações podem possuir diferentes perspectivas, mas sempre considerando como tem sido a experiência de homens e mulheres nesse momento do desenvolvimento humano e, sobretudo, na atualidade.

\section{Conclusão}

O presente estudo reafirma a importância de examinar o papel dos esquemas de gênero na constituição de quadros de estresse na adolescência. Os resultados sugerem que o perfil isoesquemático, em que existe uma simetria entre os dois esquemas de gênero (masculino ou feminino), é menos vulnerável ao estresse. Essa perspectiva é relevante quando consideramos a adolescência e suas características de desenvolvimento, não associada apenas ao sexo biológico ou aos caracteres sexuais, mas às expectativas sociais e culturais sobre como homens e mulheres devem ser, agir e existir, o que pode ser uma fonte de adoecimento.

Algumas limitações do estudo devem ser consideradas. Primeiramente, os dados foram todos baseados em 
instrumentos de autorrelato, aspecto que pode ter contribuído para as tímidas associações identificadas. Outras pesquisas podem incluir outros informantes ou ainda empregar diferentes técnicas na coleta de dados. Em segundo lugar, o delineamento transversal impede inferências causais relacionadas às variáveis em análise. Pesquisas longitudinais podem ampliar os resultados nessa direção (causa/efeito). Em terceiro lugar, a inclusão de adolescentes do Ensino Médio Profissionalizante de uma escola pública permite a generalização dos dados apenas para grupos com as mesmas características. Outras investigações podem ampliar o número amostral, bem como o tipo de instituição investigada. Por fim, os resultados se referem exclusivamente ao gênero feminino, pesquisas futuras podem incluir também o masculino.

\section{Referências}

Carvalho, P. D. e outros (2011). Condutas de risco à saúde e indicadores de estresse psicossocial em adolescentes estudantes do Ensino Médio. Cadernos de Saúde Pública. Rio de Janeiro, 27(11), 2095-2105. doi.org/10.1590/S0102-311X2011001100003

Caputo, E. L.; Rombaldi, A. J \& Silva, M. C. (2017). Sintomas de estresse pré-competitivo em atletas adolescentes de handebol. Revista Brasileira de Ciências do Esporte. Porto Alegre, 39(1), 68-72. doi.org/10.1016/j.rbce.2016.01.006

Crivelatti, M. M. B.; Durman, S. \& Hofstatter, L. M (2006). Sofrimento psíquico na adolescência. Texto \& Contexto Enfermagem. Florianópolis, 15, 64-70. doi.org/10.1590/S0104-07072006000500007

Fitzpatrick, M. J. \& Mcpherson, B. J. (2010). Coloring within the lines: gender stereotypes in contemporary coloring books. Sex Roles. Springer, 62, 127-137. doi.org/10.1007/s11199-0099703-8

Giavoni, A (2002). Estereótipos sexuais aplicados às nadadoras. Revista Brasileira de Ciência e Movimento. Brasília, 10(2), 27-32.

Giavoni, A. \& Tamayo, Á. (2005). Inventário Feminino dos Esquemas de Gênero do Autoconceito (IFEGA). Estudos de Psicologia. Natal, 10(1), 25-34. doi.org/10.1590/S1413294X2005000100004

Gonzaga, L. R. V (2013). Estresse na adolescência: problema e solução. Psicologia em Estudo. Maringá, 18(1)181-183. doi.org/10.1590/S1413-73722013000100019

Just, A. P. \& Enumo, S. R. F (2015). Problemas emocionais e de comportamento na adolescência: o papel do estresse. Boletim Paulista de Psicologia. São Paulo, 35(89), 350370. 
Kulik, L (2006). Gender, gender identity, ethnicity, and stereotyping of children's chores: The Israeli case. Journal of Cross-Cultural Psychology. SagePub, 37, 408-420. doi.org/10.1177/0022022106288477

Lee, C.-Y. S. \& Dik, B. J (2017). Associations among stress, gender, sources of social support, and health in emerging adults. Stress and Health. Wiley, 33, 378-388. doi.org/10.1002/smi.2722

Melo, G.F. \& Giavoni, A (2010). O perfil psicológico de atletas baseado na teoria do individualismo e do coletivismo. Revista Brasileira de Psicologia do Esporte. São Paulo, $3(1), 2-18$.

Melo, G.F.; Giavoni, A. \& Troccoli, B.T (2004). Estereótipos de gênero aplicados a mulheres atletas. Psicologia: Teoria e Pesquisa. Brasília, 20(3), 251-256. doi.org/10.1590/S010237722004000300006

Mezulis, A. H. e outros (2010). Gender differences in the cognitive vulnerability-stress model of depression in the transition to adolescence. Cognitive Therapy and Research. Springer, 34, 501-513. doi.org/10.1007/s10608-009-9281-7

Pinto, A. A. e outros (2017). Associação entre estresse percebido na adolescência, peso corporal e relacionamentos amorosos. Revista Paulista de Pediatria, São Paulo, 35(4), 422-428. doi.org/10.1590/1984-0462/;2017;35;4;00012

Range, L.M. \& Jenkins, S.R (2016). Who benefits from Pennebaker's expressive writing paradigm? Research recommendations from three gender theories. Sex Roles. Springer, 63, 149-164.

Sadir, M. A.; Bignotto, M. M. \& Lipp, M. E. N (2010). Stress e qualidade de vida: influência de algumas variáveis pessoais. Paidéia. Ribeirão Preto, 20(45), 3-81. doi.org/10.1590/S0103$863 \mathrm{X} 2010000100010$

Starr, C.R. \& Zurbriggen, E.L (2017). Sandra Bem's Gender Schema Theory after 34 years: a review of its reach and impact. Sex Roles. Springer, 76, 566-578. doi.org/10.1007/s11199-016$0591-4$

Tricoli, V. A. C. \& Lipp, M. E. N. (2005). Escala de Stress para Adolescentes - ESA. Porto Alegre, Sinopsy Editora.

\section{Os autores:}

Sérgio Cardoso Barcelos é mestre em Educação Física pela Universidade Metodista de Piracicaba - UNIMEP/SP. Professor de Educação Física CEFET/MG - Campus Araxá, e-mail: sergiocardosobarcelos@gmail.com

Jorge Luiz da Silva é psicólogo, doutorado em Enfermagem em Saúde Pública (USP). Docente do curso de Psicologia e pesquisador do Programa de Pós-Graduação em Promoção da Saúde da Universidade de Franca, e-mail: jorge.silva@unifran.edu.br

Wanderlei Abadio de Oliveira é psicólogo, doutor em Ciências pelo Programa de Pós-Graduação Enfermagem em Saúde Pública, da Escola de Enfermagem de Ribeirão Preto - Universidade de São Paulo (EERP/USP). 
Departamento de Psicologia da Faculdade de Filosofia, Ciências e Letras de Ribeirão Preto (FFCLRP/USP), e-mail: wanderleio@usp.br

Cléria Maria Lobo Bittar é psicóloga, doutora em Serviço Social (UNESP). Docente do curso de Psicologia e pesquisadora do Programa de Pós-Graduação em Promoção da Saúde da Universidade de Franca - SP, email: profa.cleriabittar@gmail.com

Recebido em: 26/10/2018

Aprovado em: 28/12/2018 